\title{
Applying general markup knowledge to analyze ionograms of various ionosondes
}

\author{
Vladimir Mochalov*, Anastasia Mochalova \\ Institute of Cosmophysical Research and Radio Wave Propagation of the Far Eastern Branch of \\ Russian Academy of Science, 684034, Kamchatskiy kray, Paratunka, Mirnaya str. 7, Russia
}

\begin{abstract}
In order to improve the quality of recognition of ionograms, the use of general knowledge about the reference marking of ionograms at various points of installation of ionosondes of the same type is considered. On the basis of reference markings from two ionosondes, deep neural networks were trained to highlight reflection traces from different layers of the ionosphere. The resulting deep neural networks have been successfully applied to recognize ionograms of another type of ionosonde. The results of recognition are presented.
\end{abstract}

\section{Introduction}

In works [1, 2], it is proposed to apply deep learning for recognition of ionograms and to further distinguish ionospheric parameters based on recognized layers. An outstanding feature of the new method proposed by the authors in [1] is the use of deep learning to recognize traces of reflections from different layers of the ionosphere. Deep neural network learning is realized on the basis of reference markings created by operators. Thus, ionosonde "Parus-A" [3] has been operated at the Institute of Cosmophysical Research and Radio Wave Propagation since August 2015. It records ionograms every 15 minutes. The operators, interpreting and processing ionograms, make ionogram marking at the beginning of every hour. On average, three out of four ionograms for each hour are unprocessed by the operators. About 40000 ionograms have been processed during the time of "Parus-A" functioning. They are the basis for deep neural network learning. The operators mark ionospheric parameters on the ionograms and detect, when possible, reflection traces from E, F1 and F2 layers of the ionosphere.

The problem of recognition of reflection traces from different ionospheric layers may be referred to the class of object segmentation on images. By the present time, scientists have developed different architectures for deep neural networks, which are used for object segmentation on images (for example, U-Net [4], Mask R-CNN [5], Deep Watershed Transform [6] etc.). Currently, the architecture of deep neural network U-Net has been chosen to detect the reflection traces from different ionospheric layers. In order to recognize the reflection traces from ionospheric E, F1 and F2 layers, we trained a separate deep neural network (DNN) for each layer and used black-and-white masks marked by operators. We used dice-coefficient loss (DCL) function: $D C L=1-\frac{2|X \cap Y|}{|X|+|Y|}$, where $\mathrm{X}-$ is a set

* Corresponding author: vmochalov@ikir.ru 
of ionogram layer points marked by operators and Y - is a set of detected by DNN ionogram layer points. We used 10 percent of all examples to create a validation dataset. Currently, we have obtained the following results of the DCL function evaluation for a validation dataset when compressing an ionogram to a size of $64 x 48$ pixels:

a) for DNN for F2 layer recognition DCL is 0.10842 ;

b) for DNN for F1 layer recognition DCL is 0.16890;

c) for DNN for E layer recognition DCL is 0.15796 .

It should be noted that the total number of training examples for layers F1 and E was smaller compared to layer F2, since at night layers F1 and E disappear and are not labeled by operators. To make a decision on the need to recognize traces of reflection from the F1 and $\mathrm{E}$ layers, the following rule is used: if the solar altitude at the moment of formation of the ionogram at the installation place of the ionosonde is less than the set threshold, then recognition of traces of reflection from the layers is not performed [2].

\section{Training the deep neural networks based on data from two different ionospheric stations}

In order to check how the recognition accuracy of the ionosphere layers changes when using data from various stations, data were taken from the ionosonde "Parus-A" in Paratunka in Kamchatka (40000 marked ionograms) and data from the ionosonde "Parus-A" in Moscow (74350 marked ionograms).

The following results were obtained on estimating the loss function on the validation set of ionograms when they are compressed to a size of $64 \times 48$ pixels:

a) for DNN for F2 layer recognition DCL is 0.10347 ;

b) for DNN for F1 layer recognition DCL is 0.15518;

c) for DNN for E layer recognition DCL is 0.11761 .

Thus, the recognition accuracy of the F2 layer was improved by $\sim 1.0478$ times, the recognition accuracy of the F1 layer was improved by $\sim 1.088$ times, the recognition accuracy of the E layer was improved by $\sim 1.343$ times. The results are compared when using data from two ionospheric stations (Paratunka and Moscow) with data from only one Paratunka ionospheric station.

Consider the process of layer-by-layer recognition of ionospheric layers in ionograms. We use strategy in which each layer of the ionosphere after it is recognized in the ionogram image is removed to more accurately recognition of the subsequent layers of the ionosphere. The following sequence of layer recognition was taken: first, the F2 layer is recognized and the recognized pixels of the F2 layer are removed from the analyzed image of the ionogram; then, layer $\mathrm{E}$ is recognized and the recognized pixels of layer $\mathrm{E}$ are removed from the analyzed image of the ionogram; then layer F1 is recognized. The results of applying this sequence made it possible to improve the loss function for recognizing the F1 layer, while the loss function for recognizing the F2 and E layers did not practically change. Thus, the recognition accuracy of the F1 layer was improved by $~ 1.12$ times.

\section{Using trained deep neural networks to recognize ionograms from a different type of ionosonde}

In Kamchatka (Paratunka), the PCS4 FMCW ionosonde (Figure 1) functions, which is significantly inferior in the quality of the produced ionograms in comparison with the ionosonde "Parus-A", but at the same time makes ionograms 5 times more often in time (every 3 minutes in comparison with every 15 minutes of ionosonde "Parus-A"). There are no reference markings for the PCS4 FMCW ionosonde, but there are many ionograms, the 
extracted parameters of which can be very useful for scientific research. To use ready-made trained deep neural networks for recognition of ionograms of the PCS4 FMCW ionosonde, it was necessary to convert the ionograms to a general form. Figure 2 shows an example of a PCS4 FMCW ionosonde ionogram, and Figure 3 shows a transformed view of the ionogram, which can already be used by deep neural networks to recognize reflection traces from different layers of the ionosphere. The deep neural networks described in section 2 have been successfully applied to recognize ionograms of PCS4 FMCW ionosonde. Figure 4 shows the result of the work of the ionogram analysis support system [2], which highlighted the reflection trace from the F2 layer of the ionosphere in the example under consideration. Figure 5 shows the ionogram from the "Parus-A" ionosonde, marked by the operator and using deep neural networks, which was obtained at 15.07.2020 13:00 UTC.
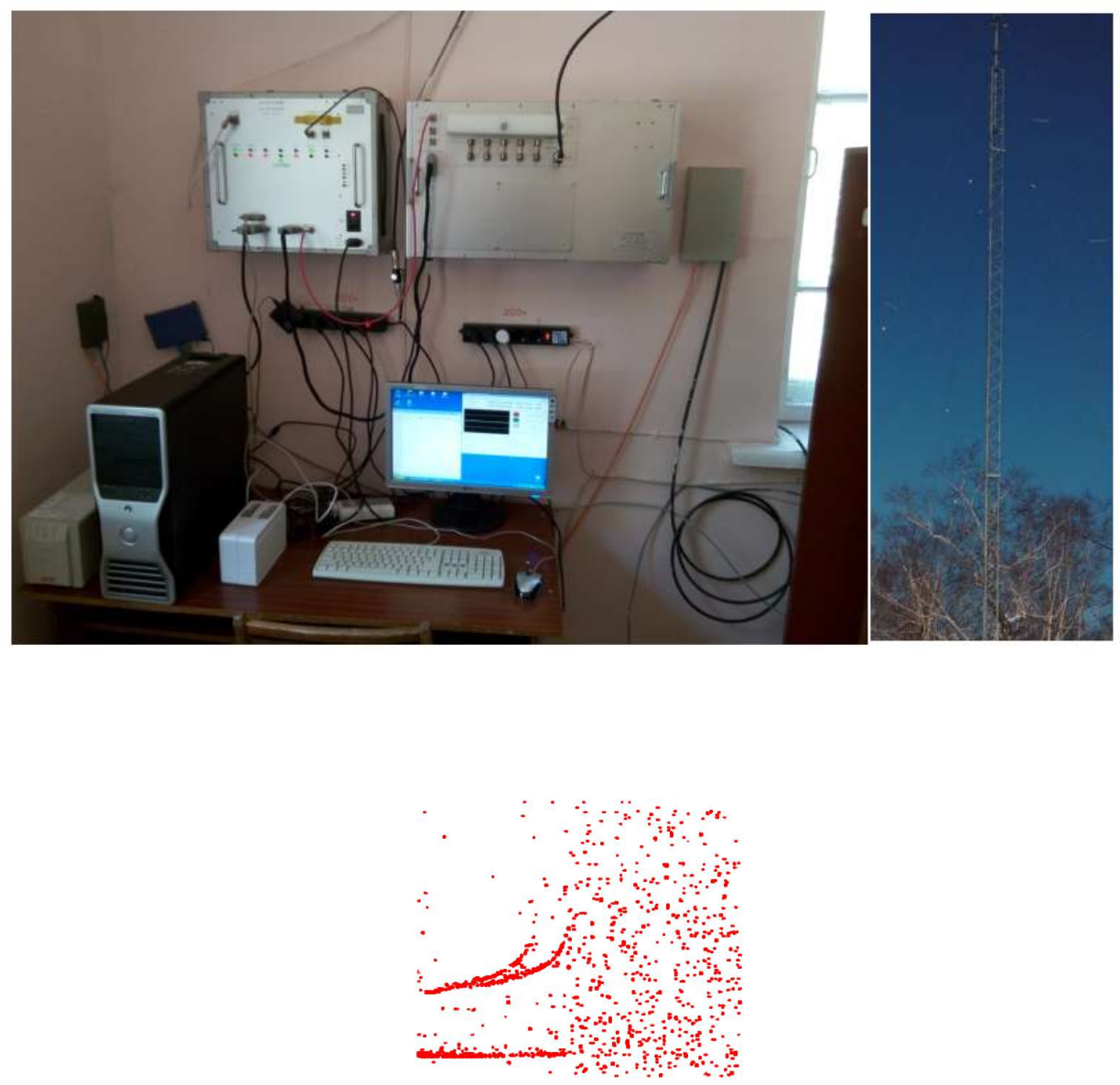

Fig. 3. The transformed view of the ionogram, which can already be used at the recognition stage.
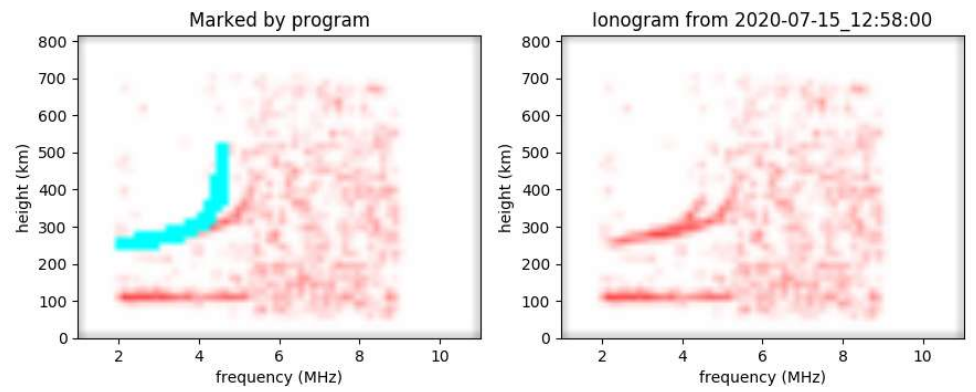
Fig. 4. Highligting of the reflection trace from the F2 layer of the ionosphere in the ionogram from the PCS4 FMCW ionosonde.
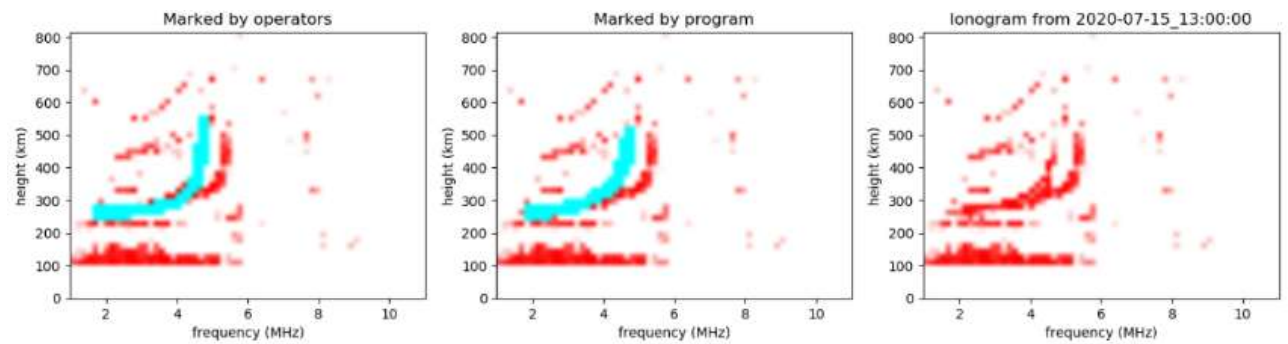

Fig. 5. Highligting of the reflection trace from the F2 layer of the ionosphere in the ionogram from the "Parus-A" ionosonde.

Figure 6 shows the marked ionogram obtained from the PCS4 FMCW ionosonde in the daytime (15.07.2020 00:04 UTC). Figure 7 shows the ionogram from the "Parus-A" ionosonde, marked by the operator and using deep neural networks, which was obtained at 15.07.2020 00:00 UTC.
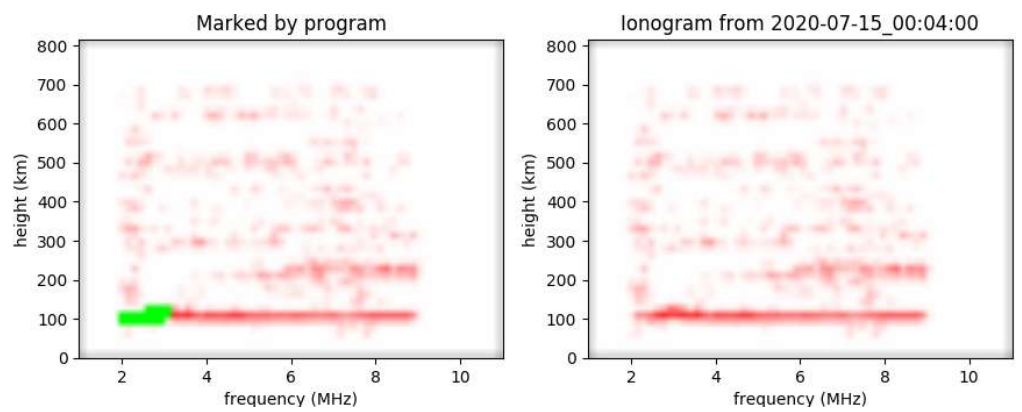

Fig. 6. Highligting of the reflection trace from the E layer of the ionosphere in the ionogram from the PCS4 FMCW ionosonde.
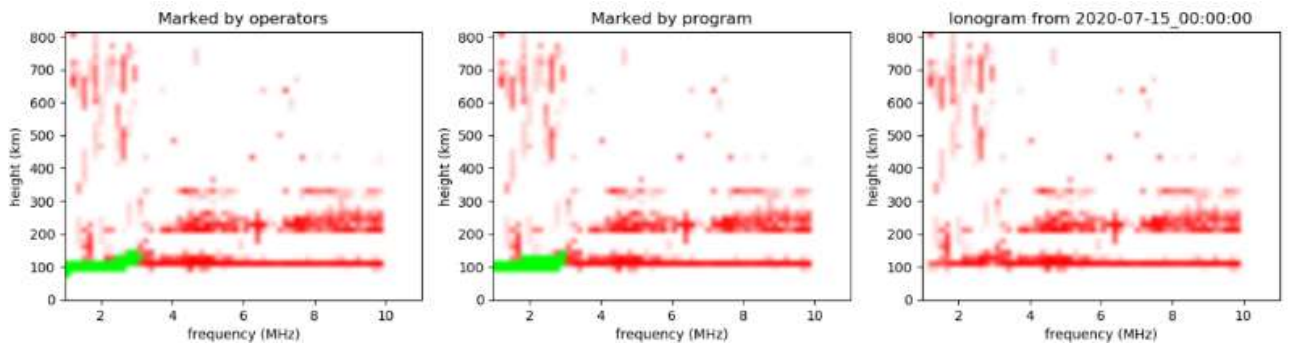

Fig. 7. Highligting of the reflection trace from the $E$ layer of the ionosphere in the ionogram from the "Parus-A" ionosonde.

\section{Conclusion}

Based on the results obtained in the work, the following conclusions can be drawn: 1. An increase in the number of used stations and training examples leads to an increase in the accuracy of recognition of the layers of the ionosphere. So, the recognition accuracy of the F2 layer was improved by 1.0478 times, the recognition accuracy of the F1 layer was 
improved by $\sim 1.088$ times, the recognition accuracy of the E layer was improved by $\sim 1.343$ times. The results are compared when using data from two ionospheric stations (Paratunka and Moscow) with data from only one Paratunka station.

2. A strategy of sequential recognition of ionospheric layers is proposed. First, the F2 layer is recognized and it is removed from the image, then the E layer is recognized and it is removed from the image, and finally the F1 layer is recognized. Thus, the recognition accuracy of the F1 layer was improved by 1.12 times.

3. Trained deep neural networks can be used to recognize ionograms from a different type of ionosonde.

4. Since the noise level in different ionosondes is different, it is important to: remove noise from ionograms and train deep neural networks with the addition of artificial noise.

5. Since there were no reference markings for the PCS4 FMCW ionosonde and it is close to the "Parus-A" ionosonde, the quality of recognition of ionograms should be further checked with expert operators on the basis of the reference markings of the "Parus-A" ionosonde.

6. Since the starting frequency of sounding is different for different ionosonde, it is recommended to train deep neural networks for a specific ionosonde.

\section{Acknowledgments}

The authors are very grateful to the expert operators involved in the processing and interpretation of ionograms, the results of which served as the basis for creating training data for the ionogram recognition system based on the use of deep learning.

The paper was carried out within the framework on the subject «Dynamics of physical processes in active zones of near space and geospheres» (AAAA-A17-117080110043-4).

\section{References}

1. V. A. Mochalov, A. V. Mochalova, 2019 Russian Open Conference on Radio Wave Propagation, RWP 2019 - Proceedings Web Conference, 8810326, (2019)

2. V. A. Mochalov, A. V. Mochalova, E3S Web Conference, 127, (2019)

3. G.V. Givishvili, I.V. Krasheninnikov, L.N. Leshchenko, Yu.M. Vlasov, A.V. Kuz'min, Heliogeophysical investigation, 4, 68-74, (2013) in Russian

4. Ronneberger O., Fischer P., Brox T. U-Net: Convolutional Networks for Biomedical Image Segmentation, URL: https://arxiv.org/abs/1505.0459

5. He K., Gkioxari G., Dollár P., Girshick R, Mask R-CNN, URL: https://arxiv.org/abs/1703.06870 .

6. Bai M., Urtasun R. Deep Watershed Transform for Instance Segmentation, URL: https://arxiv.org/abs/1611.08303 . 